\title{
Fluoro-siloxanyl Styrene Rigid Gas Permeable Contact Lens Fabric characteristics and outcomes with extended use on animal model cornea
}

\begin{abstract}
COSTIN ALEXANDRU LEASU BRANET ${ }^{1}$, SINZIANA ISTRATE ${ }^{1 *}$, LILIANA MARY VO INEA ${ }^{1}$, ENIKO VO LCEANOV ${ }^{2}$, RADU CIULUVICA $^{3}$, RALUCA IANCU ${ }^{1}$, ALEXANDRA BASTIAN ${ }^{5}$, EMIL UNGUREANU ${ }^{1}$, FLORIAN BALTA ${ }^{1}$

'University of Medicine and Pharmacy Carol Davila, Bucharest, Ophthalmology Department, 37 Dionisie Lupu Str., 020021, Bucharest, Romania

2Metallurgical Research Institute Bucharest, 39 Mehadia Str.,060543, Bucharest, Romania

3University of Medicine and Pharmacy Carol Davila, Bucharest, Anatomy Department, 37 Dionisie Lupu Str., 020021, Bucharest, Romania

${ }^{4}$ University of Medicine and Pharmacy Carol Davila, Bucharest, General Surgery Department, 37 Dionisie Lupu Str., 020021, Bucharest, Romania

EUniversity of Medicine and Pharmacy Carol Davila, Bucharest, Department of Pathology, 37 Dionisie Lupu Str., 020021, Bucharest, Romania
\end{abstract}

In this paper, we report the relation between fabric characteristics and animal model cornea modifications associated with extended use of fluoro-siloxanyl styrene rigid gas permeable contact lens. Siloxanyl styrene and fluoromethacrylate rigid gas permeable contact lens is a newly designed optical medical device used in orthokeratology with an FDA approval of continuous wear for up to 30 days. To assess the influence on the cornea an animal model was used and histological data were collected. Scanning electron microscopy (SEM) was performed on the used lenses. Our conclusions concedes that with respects of the material, doctor's slit lamp lens observation on regular basis should be considered as surface debris and lens scratches can influence the cornea in extended wear.

Keywords: siloxanyl styrene and fluoromethacrylate, rigid gas permeable contact lens, surface debris, extended wear.

Orthokeratology represents an alternative to refractive surgery in patients with refractive errors, especially children. It implies wearing a hard contact lens overnight in order to temporarily modify the shape of the cornea [1].

In orthokeratology every part of the cornea suffers important or minor changes based of their location and action of the gas permeable lens on the corneal surface. The epithelial change in orthokeratology is tissue compression centrally, thinning, and expansion in the midperiphery, thickening [2-5].

The most important orthokeratology effect, on cat's eyes, suggested by Choo and Caroline [6], is redistribution of the epithelial cells, central is decreasing the cell layers and mid-peripherally is increasing.

There are two theories about epithelial changes, first is about redistribution and second about compression [7]. Because corneal changes install very rapidly after putting the orthokeratology lens [8], the epithelial cells ability to weaken their bonds with other cells and take a new position, redistribute on the corneal surface is very less probable. The first change is compression related and possible after this phase, redistribution and hyperplasia of the epithelial cells in periphery [7]. Next to the epithelial changes, are the stromal changes. These changes are located in the mid-periphery of the cornea, outside the central zone, which is flat, result of the orthokeratology lens. In this area, only the epithelial suffers changes, without any stromal modification [2]. On the other hand, in the mid-periphery and periphery, the stromal changes are more important that the epithelial, with a swelling response [9]. Also, the proteoglycans in the deep central stroma increases [10]. On the posterior layers of the cornea, after orthokeratology treatment, may appear modification like posterior bending, the result of edema and molding [11].

Orthokeratology induce aberrations (especially spherical) result of all the reshaping of the anterior surface of the cornea [12].

Siloxanyl styrene and fluoromethacrylate rigid gas permeable contact lens is a newly designed optical medical device used in orthokeratology [13].

Lately, hard contact lenses containing fabrics of higher oxygen permeability compared to traditional polymethyl methacrylate (PMMA) were manufactured, and they are gathered under the name of rigid gas permeable contact lenses (RGP). The first RGPs were introduced in 1980s [14] and the component materials of RGPs varied during time from cellulose derivatives (e.g. CAB - cellulose acetate butyrate) to copolymers which enclose silicones (silicone acrylate), styrene derivatives and fluoropolymers (fluorsilicone-methacrylatecopolymer).

Due to their high oxygen permeability, nowadays RGP market is dominated by copolymers containing mostly silicone (meth)acrylate and fluoro (meth)acrylate.

Siloxanyl styrene and fluoromethacrylate rigid gas permeable contact lens (Menicon Z Night ${ }^{\circledR}$, Menicon, Japan) is a highly-permeable copolymer and the world's only gas permeable lens material approved by the FDA for up to 30 days continuous wear [13].

\section{Experimental part}

Materials and methods

The present prospective in vivo study, on animal model, is part of a PhD research which one of the objectives is to investigate the impact of extended use ( 45 days continuous

\footnotetext{
* email: sinziana.istrate@umfcd.ro, Phone: +040726535515. The authors contributed equally to the present work and thus are main authors.
} 
wear) of fluoro-siloxanyl styrene rigid gas permeable contact lens on the corneal tissue and on the lens material.

Given that there are certain types of reactions that cannot be simulated in vitro, it is necessary to set up the experiments on laboratory animals in order to simulate the body's reaction to synthetic materials.

The study was performed in University of medicine and Pharmacy bio base respecting Ethic Committee regulation on 6 rabbits (New Zeeland, female, 4 month of age rabbits). The animals used were supplied with food and water, they were provided accommodation in an environment which allowed them at least a minimum degree of freedom of movement, adequate for the health and welfare under the law stipulated in the European Convention for the protection of animals used in for experimental and other scientific purposes Strasbourg 18.03.1986 and later in Directive 2010/ 63 / EU.

The study protocol comprised in the placement of fluoromethacrylate-siloxanyl styrene copolymer (figure 1) rigid gas permeable contact lens (Menicon Z Night ${ }^{\text {, }}$, Menicon, Japan) on the right cornea, with blepharoraphy (using 4 separate nylon 7.0 sutures) in the same eye under general anesthesia. The blepharoraphy was continuously maintained for 6 weeks.

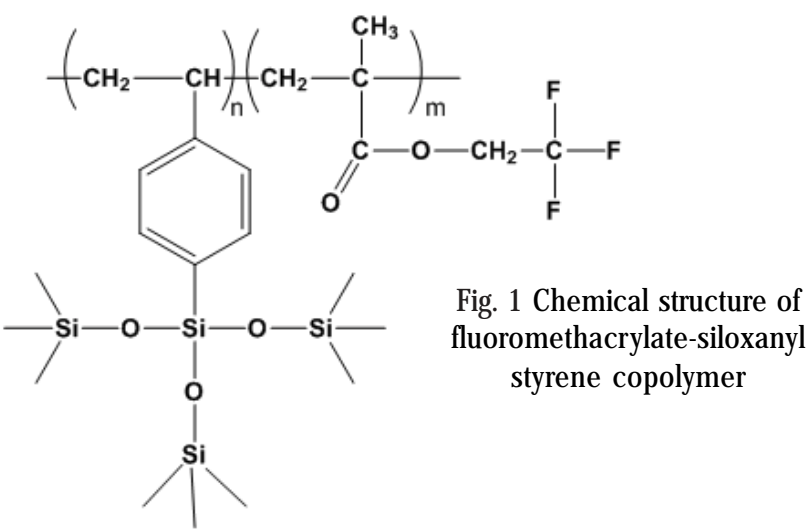

During this time rabbits received systemic broad spectrum antibiotherapy for the first week after blepharoraphy (Enrofloxacin - Ganadexil Enro 5\%, Industrial Veterinaria SA $0.5 \mathrm{ml} / \mathrm{kg} /$ day) followed by topical administration of large spectrum antibiotics (Tobrex, eye ointment Alcon bid) for another 7 days. After 45 days the blepharoraphy sutures were removed and the lens was extracted, the eyes were enucleated and the central corneas were dissected from enucleated eyeballs.

The samples were embedded in 10\% neutral-buffered formalin in order to be examined in light microscopy. Corneal sections were stained with haematoxylin and eosin. Also, immunohistochemistry was performed for platelet endothelial cell adhesion molecule-1 (CD31) for detection of corneal neovascularization. At haematoxylin eosin coloration three main corneal structures were noted: corneal epithelium, corneal stroma and corneal endothelium.

The lenses were rinsed in Menicare $₫$ (Menicon, Japan) solution and prepared for SEM (Scanning electron microscopy) examination.

\section{Results and discussions}

In 4 out of 6 rabbit's corneal structures had normal appearance, but in 2 cases corneal stroma exhibits eosinophilic infiltration and also epithelial layer proliferation (figure 2,3). Corneal epithelium alteration consisted in: disorganization and separation of cells, vacuolated cytoplasm, abnormal nuclei and superficial cells shedding. In those 2 specimens, corneal surface architecture was 4004 modified (irregular corneal surface); stromal lamellar network presented irregularities and also immune cells infiltration (eosinophils) (figure 2,3).

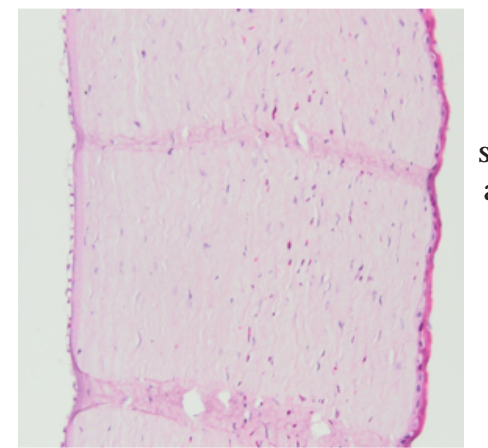

Fig. 2 CD31

Immunohistochemistry

Coloration X10. Intact superficial corneal epithelium and normal avascular corneal stroma (immunohistochemistry is negative for platelet endothelial cell adhesion molecule-1).

Fig. 3 Hematoxilin-eosin coloration X20. Intact anterior stratified squamous non-keratinising corneal epithelium, stromal mixed chronic inflammatory cell infiltrate including

eosinophils between the lamellae of the substantia propria.

Scanning electronic microscopy examination of the contact lens revealed good material aspects after extended use under closed eyelids. Two lenses, corresponding to the 2 cases with eosinophilic infiltration presented on surface organic material un-removed by normal rinsing technique and several superficial surface scratches (figure 4,5).

The present study showed minimal corneal ultrastructural changes in 2 out of 6 cases of prolonged use of fluoro-siloxanyl styrene rigid gas permeable contact lens (Menicon Z Night $\AA$, Menicon, Japan). Extended use of this particular type of contactlens could induce structural changes of corneal epithelium and stroma.

Due to the fact that orthokeratology tends to become an appealing treatment among children with refractive errors, possible side effects associated with overnight use

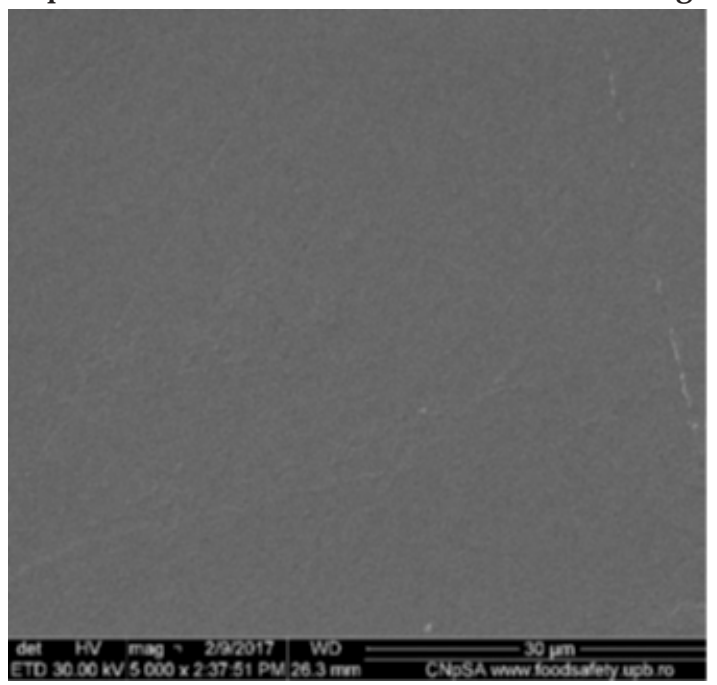

Fig. 4 SEM of the lens from the normal cornea. Several surface superficial scratches can be seen without organic material deposition 


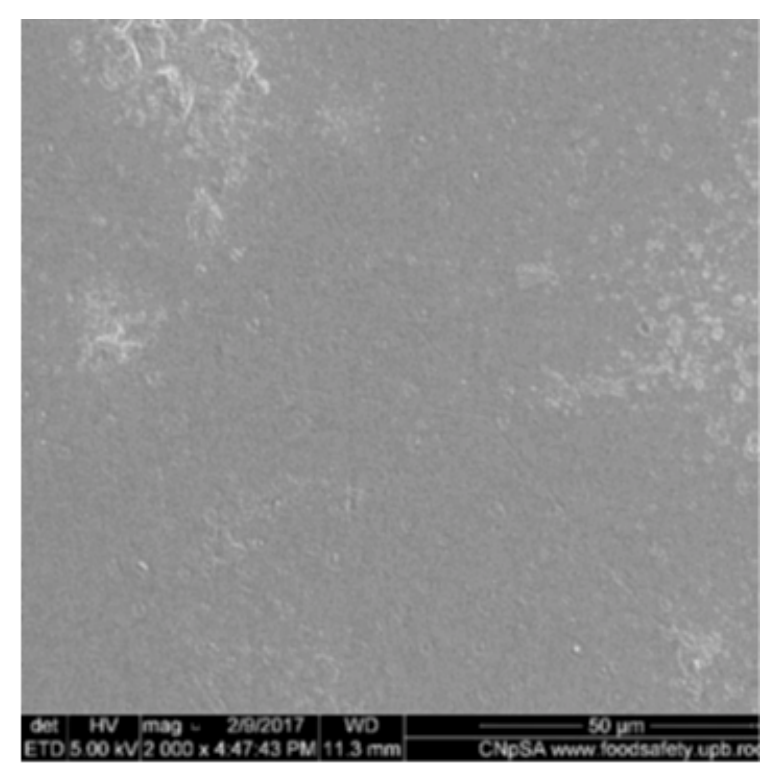

Fig. 5 SEM of the lens from the cornea with the eosinophilic infiltration. Several superficial surface scratches and surface organic material can be note

of RGPs are continuously evaluated. Considering this, RGP materials should encounter particular properties such as oxygen permeability, wettability, scratch resistance, rigidity, durability, deposit resistance. They should also be safe, efficient and FDA-approved. From 1950s there was a tremendous effort to obtain polymeric materials applicable for manufacturing contact lenses, in order to satisfy patient comfort, to obtain good outcomes in means of visual acuity, to increase materials biocompatibility and to assure minimal alteration of the corneal bio-physiological properties. Nowadays high-grade plastic polymers are used to manufacture different kinds of contact lenses.

Corneal epithelium showed in 2 cases: disorganization and separation of cells, vacuolated cytoplasm, abnormal nuclei and superficial cells shedding. These changes are related to prolonged wear and to minimal surface irregularities discovered on the lens surface in these two particular cases (figure 3).

Corneal stroma presented slight lamellar disorganization and in two cases immune cells were present. We did not detect corneal swelling. Cornea is an avascular tissue and oxygen from external sources is extremely important for maintaining a normal metabolic activity. All contact lenses reduce oxygen availability, but a contact lens worn during night (under closed eyelids) intensify this phenomenon. Oxygen deprivation induce corneal stromal swelling. In our study we did notencounter this structural modification. This may be in part because fluorosiloxanyl styrene is hyperpermeable to oxygen molecules.

Oxygen provided to the corneal tissue, when wearing a RGP contact lens, depends on two physical properties: oxygen permeability and oxygen transmissibility [15]. Oxygen permeability (Dk) in an intrinsic characteristic of the material from which the contact lens is manufactured. It is dependent on diffusion coefficient(D) and the solubility coefficient $(k)$. The diffusion coefficient describes the velocity of the gas molecules moving in the material, and the solubility coefficient defines how much gas can be dissolved into the material. Oxygen transmissibility (Dk/t) depends on the contact lens's physical characteristics and is defined by contactlens's fabric oxygen permeability (Dk) divided by contact lens thickness $(\mathrm{t})$.

There are several material dependent aspects that decide the oxygen permeability of an RGP lens, like: polymer structure, temperature and partial pressure at the anterior surface.

The RGP contact lens materials contain siloxanes (silicones) [16]. Siloxanes integrate a group of compounds with multiple applications in healthcare [17]. Siloxanes (Si-O-Si), commonly known as silicones, are chemical elements formed by covalent bounds between silicon ( $\mathrm{Si}$ ) and oxygen (0), with functional groups attached to the silicone $[18,19]$. They encounter a number of physicochemical properties (lack of chemical reactivity, thermal stability, high oxygen permeability) which make them highly biocompatible. Siloxane chains are flexible, mobile and have the ability to deform [20]. These particular aspects make siloxanes capable to permeate gasses (like oxygen). In the course of movement (due to Si-O bond flexibility) siloxane chains form free permeable channels which permit oxygen diffusion [21]. Improvement of oxygen permeability was also obtained by the addition of a fluorinated monomer to the siloxane-acrylate, which increases oxygen solubility in RGP materials.

Menicon Z Night lenses used for the experimental part of our study, are made from FluoroSiloxanyl Styrene and have a Dk of 163.

Temperature also influences oxygen permeability (the higher the temperature, the greater the Dk). 340 is the widely accepted corneal temperature in most evaluations, and represents the temperature at corneal surface with eyes opened. In orthokeratology the patient wears the RGP contact lens during the sleeping period (with eyes closed), so we can assume that corneal temperature can exceed 340.

Siloxane acrylate fabrics which incorporate fluorinated monomers bear new characteristics and upgraded surface properties compared to siloxane acrylates alone. Fluorinated materials increase surface solubility (due to improved wettability) and make the compound more permeable to oxygen; these additives reduce surface energy, increase UV resistance (protecting ocular structures), reduce friction and therefore the interactions between the tear film and the lens (reducing protein deposition on the lens surface) $[22,23]$.

\section{Conclusions}

An optimal polymer for contact lens manufacturing should have the following characteristics: good optical properties, high gas (oxygen) permeability, toughness, inactive to corneal surface microorganisms and debris. With respects of the material, careful daily care and particularly doctor's slit lamp lens observation on regular basis should be considered as surface debris and lens scratches can influence the cornea in extended wear.

\section{References}

1. LIU, Y.M., XIE, P., Eye Contact Lens, 42, no. 1, 2016, p. 35-42.

2. ALHARBI, A., SWARBRICK, H.A., Invest Ophthalmol Vis. Sci., 44, no. 6,2003 , p. 2518-23.

3. HAQUE, S., FONN, D., SIMPSON, T., JONES, L., Eye Contact Lens, 30, no. 4, 2004, p. 189-93.

4. NICHOLS, J. J., MARSICH, M.M., NGUYEN, M., BARR, J.T., BULLIMORE, M.A., Optom Vis Sci., 77, no. 5, 2000, p. 252-9.

5. SONI, P.S., NGUYEN T.T., BONANNO, J.A., Eye Contact Lens., 29, no. 3, 2003, p. 137-45.

6. CHOO, J.D., CAROLINE, P.J ., HARLIN, D.D., PAPAS, E.B., HOLDEN, Cont Lens Anterior Eye, 31, no. 1, 2008, p. 29-37.

7. VAN DER WORP, E., RUSTON, D., Optometry in Practice, 7, 2006, p. 47-60.

8. SRIDHARAN, R., SWARBRICK, H., Optom Vis Sci., 80, no. 3, 2003, p. 200-6. 
9. ALHARBI, A., LA HOOD, D., SWARBRICK, H.A., Invest Ophthalmol Vis Sci., 46, no.7, 2005, p. 2334-40.

10. MATSUBARA, M., KAMEI, Y., TAKEDA, S., MUKAI, K., ISHII, Y., ITO, S., Eye Contact Lens, 30, no. 4, 2004, p. 198-204.

11. OWNES, H., GARNER, L.F., CRAIG, J.P., GAMBLE, G., Optom Vis Sci., 81, no. 6, 2004, p. 421-6.

12. JOSLIN, C.E., WU, S.M., MCMAHON, T.T., SHAHIDI, M., Optom Vis Sci., 80, no. 12, 2003, p. 805-11.

13.*** https://www.menicon.com/pro/our-products/gp-lens/meniconz-night/, accesed in December 2018.

14. HOLDEN, B.A., SWEENEY, D.F., LA HOOD, D., KENYON, E., Curr Eye Res., 7, no. 1, 1988, p. 49-53.

15. CAVANAGH, H.D., Eye Contact Lens, 29, no .1, 2003, p. 14-16.

16.CHOJ NOW SKI, J., CYPRYK, M.,Synthesis of linear polysiloxanes, JONES, R. G., WATARU, A., CHOJ NOWSKI, J., Silicon-Containing Polymers, the Science and Technology of Their Synthesis and Applications, Dordrecht: Kluwer Academic Publishers, 2000, p. 1-43.
17.***MOJ SIEWICZ-PIENKOWSKA, K., JAMROGIEWICZ, M., SZYMKOWSKA, K., KRENCZKOWSKA, D., Frontiers in Pharmacology; 7, 2016, p.132.

18. BUTTS, M., CELLA, J.A., WOOD, C.D., GILLETTE, G., KERBOUA, R., LEMAN, J., LEWIS, L., RAJARAMAN, S., RUBINSZTAJ N, S., SCHATTENMANN, F., STEIN, J ., WENGROVIUS, J ., WICHT, D., Silicones, In: Encyclopedia of Polymer Science and Technology, WileyInterscience, 2002, p. 765-841.

19. REFOJ O, M.F., Ann Ophthalmol., 16, no. 11, 1984, p. 1009-13. 20. MARK, J.E., Accounts of Chemical Research, 37, no. 12, 2004, p. 946-953.

21. KURIAN, P., KASIBHATLA, B., DAUM, J., BURNS, C.A., MOOSA, A., ROSENTHAL, K.S., Biomaterials, 24, 2003, p. 3493-3503.

22. J ONES, B., J CT CoatingsTech, 5, 2008, p.44-48.

23. TANG, C., LIU, W., MA, S., WANG, Z., HU, C., Progress in Organic Coatings, 69, no.4, 2010, p.359.

$\overline{\text { Manuscript received: } 3.05 .2018}$ 